\title{
IIness pattern and the relationship between the prevalence of malaria and other infections in Niger Delta University
}

\author{
${ }^{1}$ Madukosiri C.H and ${ }^{2}$ Bawo D. S \\ 1Department of Biochemistry, Niger Delta University Wilberforce Island, Amassoma Bayelsa, \\ Nigeria, W. Africa. \\ ${ }^{2}$ Department of Biological Sciences, Niger Delta University, Wilberforce Island, Amassoma, \\ Bayelsa, West Africa \\ ABSTRACT
}

\begin{abstract}
The illness pattern and the relationship between malaria and other infections were examined in the present work - a base-line information prior to a meaningful healthcare policy formulation. A period of 5 years (2007 - 2011) illness pattern was retrospectively obtained from the University Medical Centre, while questionnaires addressing the various health-related activities were administered to 500 volunteer subjects - students and staff of the institution. Biochemical parameters in the various disease conditions were determined using standard tests, while the means of frequencies of disease occurrence were compared using statistical package for social sciences (SPSS). Results of mean comparison showed that the mean of frequencies of malaria infection was significantly increasing $(p<0.05)$. On the other hand, differences in the means of serum elements and other biochemical parameters measured in the various disease states were not statistically significant $(p>0.05)$. Malaria infection was the most prevalent illness with the highest frequency occurring around the month of April, accounting for $63.83-67.10 \%$ of the illnesses compared. This was followed by upper respiratory tract infection (URTI), peptic ulcer, and candidiasis, in that order. The relationship between the illness types showed that a positive correlation existed between malaria and typhoid, and malaria and URTI, $(p<0.05)$. From the results of questionnaire analysis, $70.89 \%$ of the male students' group admitted having a frequency of one to twelve attacks of malaria infection per annum with $43.67 \%$ of them who claimed to have suffered previous typhoid infections. As a result, $25.32 \%$ were absent from lectures for a period of 1- 30 days per annum. These results showed that malaria infection was on the increase and apart from the likelihood of impacting negatively on students' performance, could represent a huge source of financial drain on a developing economy.
\end{abstract}

Keywords: illness pattern, malaria, typhoid fever, infections.

\section{INTRODUCTION}

Malaria is a vector-borne infectious disease caused by protozoan parasites of the genus Plasmodium $(P)$. Four types infect man and they include $P$. faciparum, $P$. vivax, $P$. ovale, and $P$. malaria. The former is reported to be responsible for about $80 \%$ of all malaria cases and $90 \%$ of mortality rate attributed to malaria, (Breman et at., 2004).

The present study was undertaken in order to determine the prevalence of malaria and the illness pattern in a Nigerian university in the south-south part of the country. The illness data were based on the diagnosis made on the students and staff who were ill and who reported at the university medical centre for treatment within the 5years study period - 2007 to 2011. The illnesses diagnosed include malaria, upper respiratory tract infection (URTI), peptic ulcer (P.ulcer), helminthiasis, pelvic inflammatory disease (PID), musculo-skeletal pain (MSP), headache, vaginal candiadiasis (V. candidiasis), typhoid fever, severe hypertension, gastroenteritis, allergy, furuncles, abdominal colic, vomiting and stooling, uterine fibroid, asthma, streptococcal skin infection, dermatitis, exhaust burns, conjunctivitis, snake bite, joint failure, waist pain, otitis media, diabetes mellitus, dysmenorrhoea, allergic rhinitis, eczema, appendicitis, scabies, osteoarthritis, hyperprolactinaemia, hemorrhoids, cervical lymphadenitis, urethritis, dental caries, eye defect, anxiety disorder and scabes. Data analysis concentrates on the illnesses with the higher frequencies of occurrence.

Matched with the illness pattern was the assessment of health -related activities of 500 volunteer subjects (students and staff) aged 14-60years. The health related activities were assessed through the use of questionnaires which addressed activities such as usage of self medication, mode of treatment - such as orthodox, traditional or herbal means, and type of prophylaxis - an example is the use of insecticide treated nets (ITN), types of drugs and insecticide usage. Others were absenteeism from duties or 
classes due to malaria illness, presence of environmental factors that encourage mosquito breeding, such as swamps, bushes, and open drainages, were determined.

From the questionnaires, the percentage of subjects who sought for medical attention when ill was determined. The ratio was in-turn used in the determination of the number of population likely to be affected under each parameter.

Also the level of interaction among the mineral elements, as well as those of the other metabolites in intermediary metabolism was assessed through the use of statistical analysis - correlation/regression coefficient. The intention was to enable a prediction of mineral interaction in the various illness types. Lastly it was also intended to provide evidence-based advice on public policy, as this would minimize losses that occur as a result of under/inadequate-spending in the health-care sector.

\section{METHODS}

The materials used for the present study were of three categories, retrospective data, questionnaires and chemicals for biochemical parameters.

Retrospective Data / Questionnaires: The retrospective illness data were collated from the university medical centre, for the period 2007 - 2011. The questionnaires addressing the health-related activities were administered to 500 , (out of 14,171 ) students and staff, (in accordance with the sampling method explained by Baridan, 1990), of the university who voluntarily joined the study. However at the end about 143 subjects absconded leaving us with 357 of them who continued. Finally clinical tests were carried out in order to determine the relationship between some biochemical parameters and various illnesses.

Biochemical Tests: Fifty volunteer subjects were grouped into five categories, the control, the diabetic, hypertensive, typhoid, and the malaria groups.

About $5 \mathrm{ml}$ whole blood was collected from each subject after obtaining their informed consent in accordance with the ethical standard of the local medical centre. The blood samples were allowed to clot and then spun for about 10-15 minutes in a centrifuge in order to collect the serum. The electrolyte levels were determined by electrochemical method using ion selective electrode for sodium, calcium, potassium, chloride and bicarbonate. Others, namely inorganic phosphate as phosphorus, cholesterol, bilirubin, albumin, creatinine and uric acid were determined calorimetrically.

The data generated were analyzed using statistical package for social sciences (SPSS). The association between illnesses was determined using Pearson correlation, while mean comparison was done using ANOVA (analysis of variance).

\section{RESULT}

The present study has assessed the illness pattern and the biochemical parameters in the various diseases in a university population in the south-south part of Nigeria.

Illness Pattern from Year 2007 - 2011: The result of illness pattern showed that malaria, URTI, peptic ulcer and V. candidiasis were the most prevalent and account for $63.83-67.14,10.58-19.23,4.99$ - 7.02 and $2.80-4.58(\%)$ respectively for the cases reported (tables 1-5; figs 1-5).

Table 1. Descriptive Statistics for Monthly IIIness Pattern in Year 2007

Statistics for 12 monthly frequencies of illness

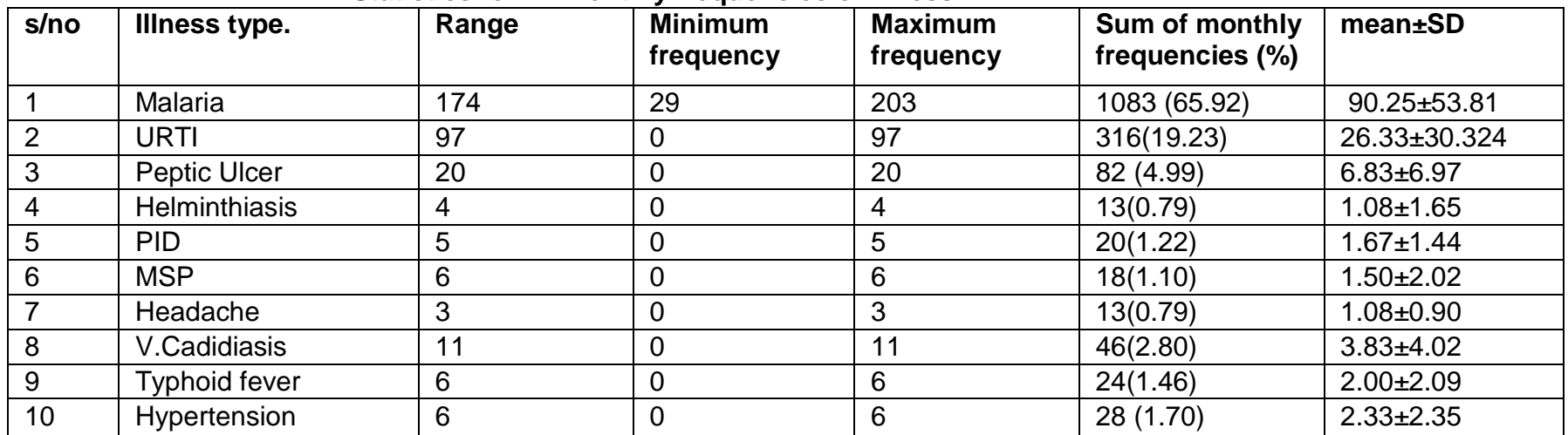

URTI, upper respiratory tract infection; PID, Pelvic inflammatory disease; MSP, Musculoskeletal pain; and V. Candidiasis, vaginal candidiasis. 
Table 2 Descriptive Statistics For Monthly IIIness Pattern For Year 2008

statistics for 12 monthly frequencies of illness

\begin{tabular}{|c|c|c|c|c|c|c|}
\hline s/no & Illness type & Range & $\begin{array}{l}\text { Minimum } \\
\text { frequency }\end{array}$ & $\begin{array}{l}\text { Maximum } \\
\text { frequency }\end{array}$ & $\begin{array}{l}\text { Sum of } \\
\text { frequencies (\%) }\end{array}$ & mean $\pm S D(\delta n-1)$ \\
\hline 1 & Malaria & 274 & 30 & 304 & 1326(65.06) & $110.50 \pm 82.22$ \\
\hline 3 & P.Ulcer & 40 & 0 & 40 & $126(6.18)$ & $10.50 \pm 12.59$ \\
\hline 4 & V. Candidiasis & 30 & 0 & 30 & $81(3.98)$ & $6.75 \pm 9.13$ \\
\hline 5 & G. Enteritis & 16 & 0 & 16 & $28(1.37)$ & $2.33 \pm 4.40$ \\
\hline 7 & Allergy & 20 & 0 & 20 & $46(2.26)$ & $3.83 \pm 5.81$ \\
\hline 8 & Abdominalcalic & 14 & 0 & 14 & $32(1.57)$ & $2.67 \pm 4.14$ \\
\hline 9 & Vomiting/ stooling & 12 & 0 & 12 & $39(1.91)$ & $3.25 \pm 3.96$ \\
\hline 10 & Boil & 15 & 0 & 15 & $26(1.28)$ & $2.17 \pm 4.61$ \\
\hline 11 & Tonsillitis & 8 & 0 & 8 & $24(1.18)$ & $2.0 \pm 2.70$ \\
\hline
\end{tabular}

URTI, upper respiratory tract infection; $P$ ulcer, peptic ulcer; V. Candidiasis, vaginal candidiasis; and G enteritis, gastroenteritis.

Table 3 Descriptive statistics for monthly illness pattern for year 2009

Statistics for 12 monthly frequencies of illness

\begin{tabular}{|l|l|l|l|l|l|l|}
\hline s/no & Ilness type. & Range & $\begin{array}{l}\text { Minimum } \\
\text { frequency }\end{array}$ & $\begin{array}{l}\text { Maximum } \\
\text { frequency }\end{array}$ & $\begin{array}{l}\text { Sum of monthly } \\
\text { frequencies }(\%)\end{array}$ & mean $\pm S D(\delta n-1)$ \\
\hline 1 & Malaria & 362 & 40 & 402 & $1600(66.47)$ & $133.33 \pm 115.04$ \\
\hline 2 & URTI & 121 & 1 & 122 & $313(13.00)$ & $26.08 \pm 32.26$ \\
\hline 3 & P.Ulcer & 62 & 0 & 62 & $169(7.02)$ & $14.08 \pm 16.87$ \\
\hline 4 & Typhoid fever & 16 & 0 & 16 & $34(1.41)$ & $2.83 \pm 5.20$ \\
\hline 5 & Hypertension & 15 & 0 & 15 & $61(2.53)$ & $5.08 \pm 4.98$ \\
\hline 6 & Boil & 30 & 0 & 30 & $41(1.73)$ & $3.42 \pm 8.67$ \\
\hline 7 & V. candidiasis & 51 & 0 & 51 & $98(4.07)$ & $8.17 \pm 14.46$ \\
\hline 8 & Allergy & 25 & 0 & 25 & $54(2.24)$ & $4.50 \pm 7.29$ \\
\hline 9 & Abdominal pain & 10 & 0 & 10 & $23(0.96)$ & $1.92 \pm 3.18$ \\
\hline 10 & Skin infection & 4 & 0 & 4 & $14(0.58)$ & $217 \pm 1.59$ \\
\hline
\end{tabular}

URTI, upper respiratory tract infection; P.Ulcer, peptic ulcer; and V. Candidiasis, vaginal candidiasis.

Table 4 Descriptive statistics for monthly illness pattern for year 2010

Statistics for 12 monthly frequencies of illness

\begin{tabular}{|l|l|l|l|l|l|l|}
\hline s/no & IIIness type. & Range & $\begin{array}{l}\text { Minimum } \\
\text { frequency }\end{array}$ & $\begin{array}{l}\text { Maximum } \\
\text { frequency }\end{array}$ & $\begin{array}{l}\text { Sum of monthly } \\
\text { frequencies (\%) }\end{array}$ & mean \pm SD \\
\hline 1 & Malaria & 539 & 23 & 562 & $1422(67.14)$ & $118.50 \pm 149.53$ \\
\hline 2 & URTI & 39 & 0 & 39 & $224(10.58)$ & $18.67 \pm 11.72$ \\
\hline 3 & P.Ulcer & 19 & 0 & 19 & $59(2.79)$ & $4.92 \pm 5.82$ \\
\hline 4 & Typhoid fever & 60 & 0 & 60 & $147(6.94)$ & $12.25 \pm 15.69$ \\
\hline 5 & V.candidiasis & 30 & 0 & 30 & $97(4.58)$ & $8.08 \pm 5.58$ \\
\hline 6 & Hypertension & 21 & 0 & 21 & $61(2.88)$ & $5.08 \pm 5.44$ \\
\hline 7 & Furuncle & 21 & 0 & 21 & $30(1.42)$ & $2.50 \pm 6.38$ \\
\hline 8 & MSP & 8 & 0 & 8 & $18(0.85)$ & $1.50 \pm 2.94$ \\
\hline 9 & PID & 12 & 0 & 9 & $16(0.76)$ & $1.33 \pm 3.14$ \\
\hline 10 & Depression & 12 & 0 & 10 & $15(0.71)$ & $1.25 \pm 2.93$ \\
\hline 11 & Helminthiasis & 12 & 0 & 10 & $15(0.71)$ & $1.25 \pm 2.93$ \\
\hline 12 & Abdominal colic & 12 & 0 & 7 & $14(0.66)$ & $1.17 \pm 2.29$ \\
\hline
\end{tabular}

URTI, upper respiratory tract infection; PID, Pelvic inflammatory disease; MSP, Musculoskeletal pain; and V. Candidiasis, vaginal candidiasis. 
Table 5 Descriptive statistics for monthly illness pattern for year 2011

Statistics for 12 monthly frequencies of illness

\begin{tabular}{|l|l|l|l|l|l|l|}
\hline s/no & Illness type & Range & $\begin{array}{l}\text { Minimum } \\
\text { frequency }\end{array}$ & $\begin{array}{l}\text { Maximum } \\
\text { frequency }\end{array}$ & $\begin{array}{l}\text { Sum of monthly } \\
\text { frequencies (\%) }\end{array}$ & Mean( \pm SD) \\
\hline 1 & Malaria & 643 & 45 & 688 & $1969(63.83)$ & $164.08 \pm 179.06$ \\
\hline 2 & URTI & 147 & 0 & 147 & $429(13.91)$ & $35.75 \pm 39.25$ \\
\hline 3 & Typhoid fever & 18 & 0 & 18 & $41(1.33)$ & $3.42 \pm 5.78$ \\
\hline 4 & PID & 10 & 0 & 10 & $26(0.84)$ & $2.17 \pm 3.74$ \\
\hline 5 & V.candidiasis & 54 & 0 & 54 & $106(3.44)$ & $8.83 \pm 15.40$ \\
\hline 6 & P.Ulcer & 85 & 0 & 85 & $245(7.94)$ & $20.42 \pm 22.79$ \\
\hline 7 & Hypertension & 23 & 0 & 23 & $62(2.01)$ & $5.17 \pm 6.70$ \\
\hline 8 & Allergy & 30 & 0 & 30 & $64(2.08)$ & $5.33 \pm 8.55$ \\
\hline 9 & Headache & 11 & 0 & 11 & $27(0.88)$ & $2.25 \pm 4.22$ \\
\hline 10 & Foruncle & 20 & 0 & 20 & $20(0.65)$ & $1.67 \pm 5.77$ \\
\hline 11 & Stomatitis & 16 & 0 & 16 & $16(0.52)$ & $1.33 \pm 4.62$ \\
\hline 12 & MSP & 29 & 0 & 29 & $80(2.59)$ & $6.67 \pm 8.75$ \\
\hline
\end{tabular}

URTI, upper respiratory tract infection; P.Ulcer, peptic ulcer; PID, Pelvic inflammatory disease; and V. Candidiasis, vaginal candidiasis.

Questionnaire Analysis: From the questionnaires (addressing the various health-related activities), about $30.43-31.58 \%$ of the female students' group claimed they visited the health centers whenever they felt ill of malaria, whereas $8.69-18.42 \%$ resorted to self medication, out of which 56.52 - $64.04 \%$ recovered after treatment. About 88.60 - 91.30\% claimed to use orthodox treatment. On the other hand, responses on prophylaxis activities showed that $84.21-100 \%$ of respondents knew about ITN (insecticide treated Nets), but only $13.04-42$. 98\% of them actually made use of the nets. Even among users, most of them never re-impregnated nets with insecticides throughout the period of use. About $13.04-19.30 \%$ claimed they used prophylactic drugs against malaria, whereas, $68.42-69.57 \%$ used other means of prevention such as the use of insecticides. Presence of mosquitoes breeding places followed the order water-logged areas $>$ open drainages > swampy areas $>$ bushy areas. About $56.52-62.28 \%$ of the female students admitted having about one to eight bouts of malaria attack per annum, consequently $13.04-25.44 \%$ were absent from classes for one to seven days annually. The male counterparts were more affected (Tables 6-7).

Biochemical Parameters in Serum: The relationship between serum biochemical parameters within groups, showed positive correlation between bicarbonate and chloride, sodium and potassium, inorganic phosphate and calcium, bilirubin and creatinine, and uric acid and chlolesterol, in the control, malaria, typhoid, diabetes, and hypertensive groups respectively, $(P<0.05)$. On the other hand student's t-test showed no significant statistical difference $(P>0.05)$ between the means $( \pm S D)$ of the analytes, (tables 10-11)

Determination of Relationship between IIIness Patterns by use of Correlation coefficient and ANOVA: The result of inter-relationship between illness pattern showed that correlation coefficient determined between malaria and typhoid fever in the year 2007 was not significant $r=-0.245 \quad(P>0.05)$, whereas in 2009, 2010 and 2011, significant correlation was obtained $(p<0.05)$. There was no typhoid fever case reported at the medical centre in 2008.

On the other hand result of mean comparison of frequencies of malaria illnesses in the 5 years period showed, with the exception of that of 2007 and 2010, no significant differences, $(P>0.05)$. Also no significant difference was obtained in the mean frequencies of URTI $(\mathrm{P}<0.05)$ during the same period. 
Agric. Biol. J. N. Am., 2012, 3(10): 413-426

Table 6: Health related activities undertaken by female students age group 11-30years

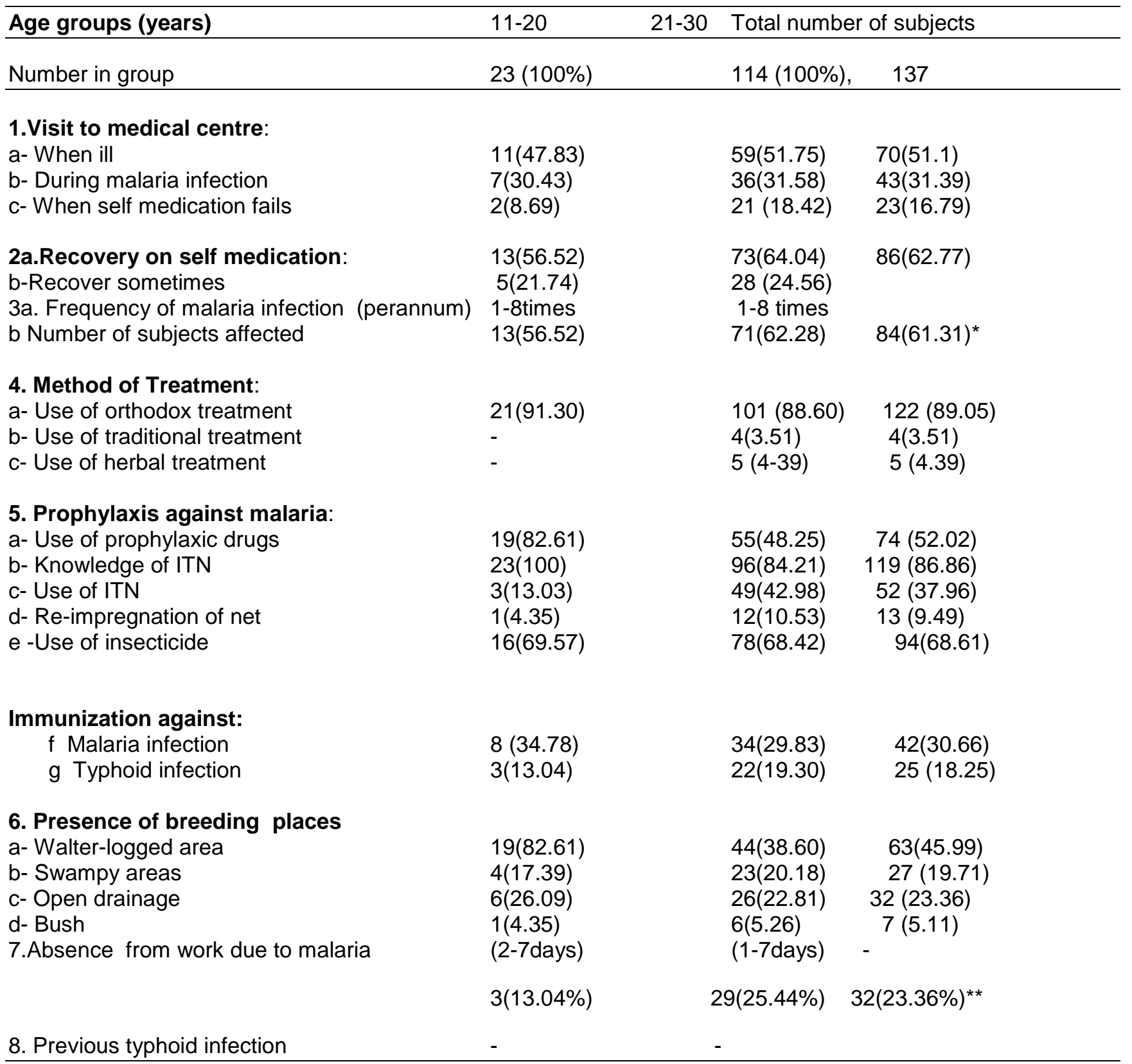

About $62.31 \%{ }^{*}$ of female students had malaria attack one to eight times per annum; while $23.36 \%{ }^{* *}$ stayed off classes for 1-7 days due to malaria illness. 
Agric. Biol. J. N. Am., 2012, 3(10): 413-426

Table 7 Health related activities undertaken by male students 11-40years

Parameter Age groups (years) Total number Total

\begin{tabular}{|c|c|c|c|c|}
\hline \multirow[b]{2}{*}{ Number of subjects in groups(\%) } & \multirow{2}{*}{$\frac{11-20}{27(\%)}$} & \multirow{2}{*}{$\begin{array}{l}21-30 \\
122(\%)\end{array}$} & \multicolumn{2}{|l|}{$31-40$} \\
\hline & & & $9(\%)$ & $158(100 \%)$ \\
\hline $\begin{array}{l}\text { I Visit to medical centre: } \\
\text { a-When ill } \\
\text { b-During malaria infection } \\
\text { c-When other medications fail }\end{array}$ & $\begin{array}{l}10(37.04) \\
8(29.63) \\
8(29.63)\end{array}$ & $\begin{array}{l}64(52.46) \\
35(28.69) \\
20(16.39)\end{array}$ & $\begin{array}{l}1(11.11) \\
3(33.33) \\
3(33.33)\end{array}$ & $\begin{array}{l}7(47.47 \%) \\
46(29.11) \\
31(19.62)\end{array}$ \\
\hline $\begin{array}{l}\text { 2a. Recovery on self medical } \\
\text { 3a. Frequency of malaria infection } \\
\text {-Number of subjects affected }\end{array}$ & $\begin{array}{l}20(74.07) \\
1-6 \text { times } \\
13(48.15)\end{array}$ & $\begin{array}{l}90(73.77) \\
1-6 \text { times } \\
93(76.23)\end{array}$ & $\begin{array}{l}7(77.78) \\
3-12 \text { times } \\
6(66.67)\end{array}$ & $\begin{array}{r}117(74.05) \\
112(70.89)\end{array}$ \\
\hline $\begin{array}{l}\text { 4. Method of treatment } \\
\text { a- Use of orthodox treatment } \\
\text { b- Use of traditional treatment } \\
\text { c- Use of herbal treatment }\end{array}$ & $\begin{array}{l}19(70.37) \\
2(7.41) \\
3(11.11)\end{array}$ & $\begin{array}{l}93(76.23) \\
7(5.74) \\
12(9.84)\end{array}$ & $\begin{array}{l}6(66.67) \\
1(11.11) \\
2(22.22)\end{array}$ & $\begin{array}{l}118(74.68) \\
10(6.33) \\
17(10.76)\end{array}$ \\
\hline $\begin{array}{l}\text { 5. Prophylaxic against malaria: } \\
\text { a- Use of prophylaxic drugs } \\
\text { b- Knowledge ITN } \\
\text { c- Use of ITN } \\
\text { d- Re-impregnation of net } \\
\text { Immunization against: } \\
\text { e- Malaria infection } \\
\text { f. Typhoid fever } \\
\text { g- Use of insecticide }\end{array}$ & $\begin{array}{l}12(44.44) \\
22(81.48) \\
5(18.52) \\
0(090) \\
12(44.44) \\
3(11.11) \\
16(59.26)\end{array}$ & $\begin{array}{l}49(40.16) \\
96(78.69) \\
47(38.52) \\
18(14.75) \\
30(24.59) \\
21(71.21) \\
69(56.56)\end{array}$ & $\begin{array}{l}4(44.44) \\
7(77.78) \\
3(33.33) \\
1(11.11) \\
\\
2(22.22) \\
1(11.11) \\
5(55.56)\end{array}$ & $\begin{array}{c}65(4.14) \\
125(79.11) \\
55(34.81) \\
19(12.03) \\
\\
44(27.85) \\
25(15.82) \\
90(56.96)\end{array}$ \\
\hline $\begin{array}{l}\text { 6. Presence of Breeding Places } \\
\text { a- Swatter-logged } \\
\text { b- Swamp } \\
\text { c- Open drainage }\end{array}$ & $\begin{array}{l}6(22.22) \\
4(14.82) \\
17(62.96)\end{array}$ & $\begin{array}{l}46(37.70 \\
24(19.67) \\
38(31.15)\end{array}$ & $\begin{array}{l}4(44.44) \\
3(33.33) \\
2(22.22)\end{array}$ & $\begin{array}{l}56(35.44) \\
31(19.62) \\
57(36.08)\end{array}$ \\
\hline d- Bushy areas & $2(7.41)$ & $6(4.92)$ & $1(11.11)$ & $9(5.70)$ \\
\hline \multicolumn{2}{|c|}{7 Absence from work due to malaria $1-16$ days } & 2-14days & 7-30days & \\
\hline & $8(29.63)$ & $29(15.57)$ & $3(33.33)$ & $40(25.32)$ \\
\hline 8.Previous typhoid infection & $14(51.85)$ & $51(41.80)$ & $4(44.44)$ & $69(43.67)$ \\
\hline
\end{tabular}

About $48.15 \%$ of respondents (age group 11-20years) had malaria attack at the rate of one to six times a year, while $29.63 \%$ stayed off classes for one to sixteen days. 
Agric. Biol. J. N. Am., 2012, 3(10): 413-426
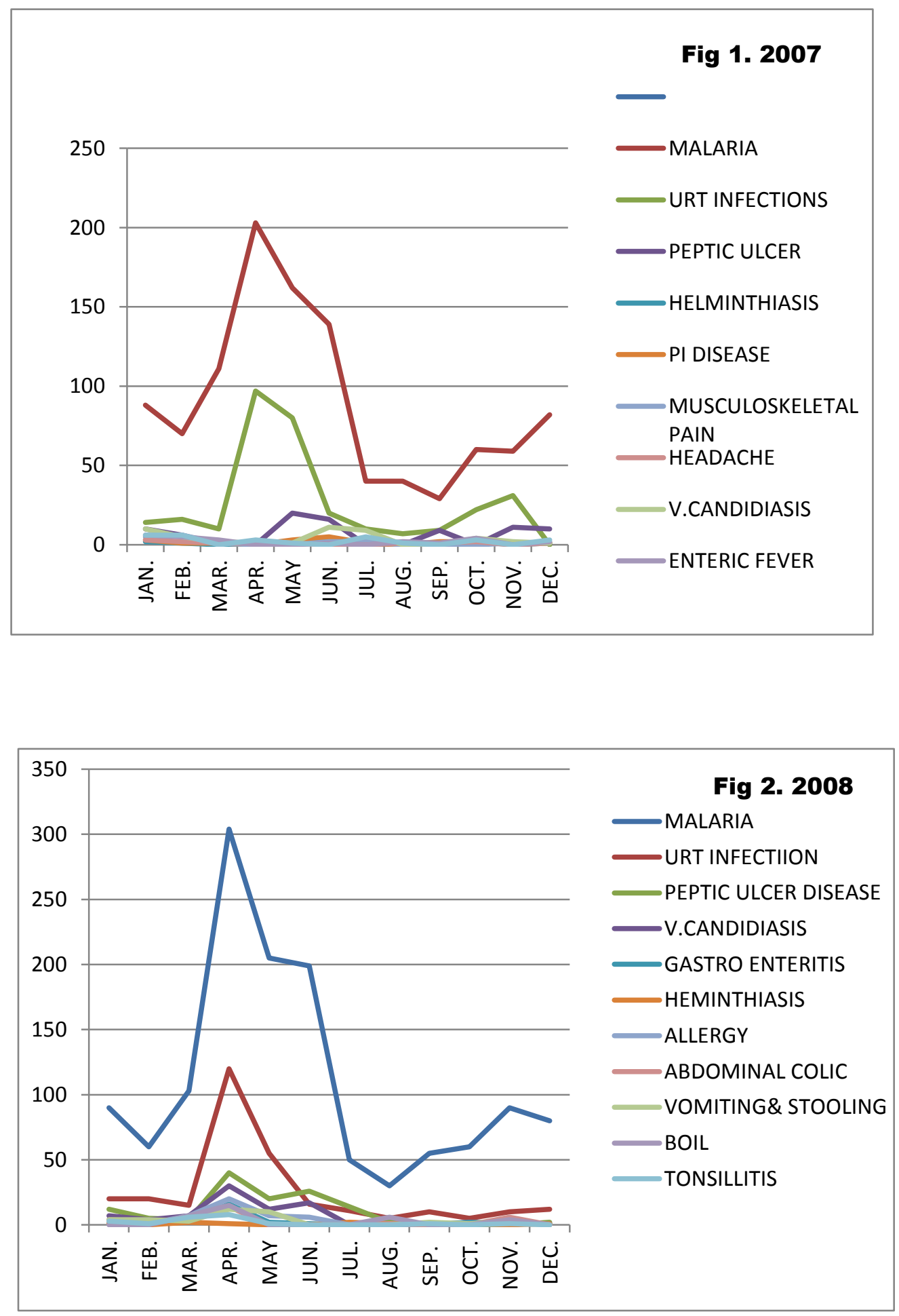

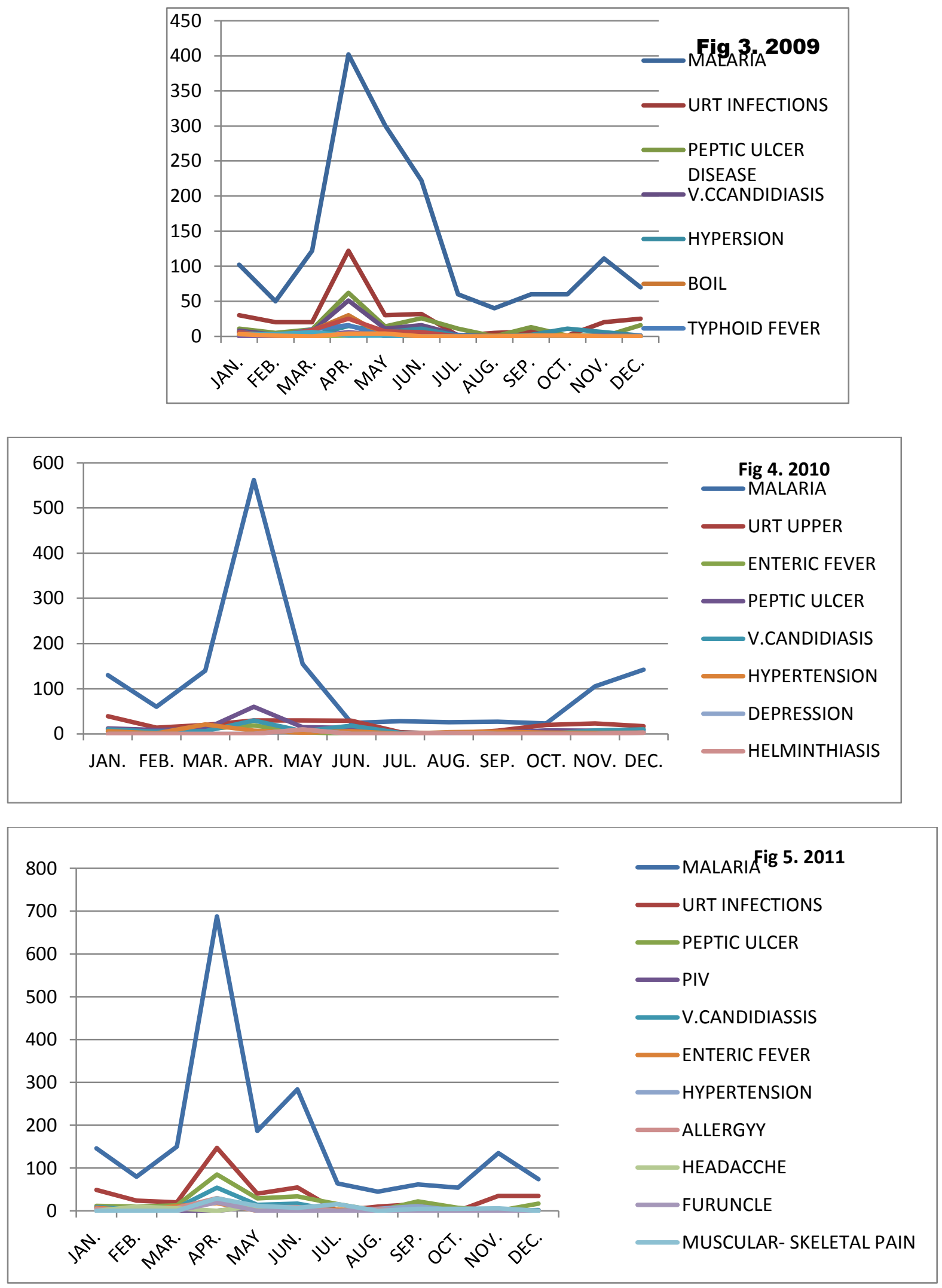
Agric. Biol. J. N. Am., 2012, 3(10): 413-426

Table 8: health related activities undertaken by female staff

Parameter

AGE GROUPS 21-60 YEARS

\begin{tabular}{|c|c|c|c|c|c|c|}
\hline \multicolumn{2}{|c|}{ No of subjects in group (\%) } & \multirow{2}{*}{$\begin{array}{l}21-30 \\
4(100 \%) \\
3(75) \\
1(25) \\
-\end{array}$} & \multirow{2}{*}{$\begin{array}{l}31-40 \\
8(100 \%) \\
4(50) \\
3(35.5) \\
0 \%\end{array}$} & \multirow{2}{*}{$\begin{array}{l}41-50 \\
8(100 \%) \\
3(37.5) \\
0 \% \\
2(25)\end{array}$} & \multirow{2}{*}{$\begin{array}{l}51-60 \\
5(100 \%) \\
3(60) \\
3(60)\end{array}$} & \multirow{2}{*}{$\begin{array}{l}\text { Total } \\
25 \\
13(25 \%) \\
6(24 \%) \\
2(8 \%)\end{array}$} \\
\hline 1 & $\begin{array}{l}\text { Visit to medical centre: } \\
\text { a-When ill } \\
\text { b- During malaria infection } \\
\text { c- When other medication fails }\end{array}$ & & & & & \\
\hline $\begin{array}{l}2 \\
3\end{array}$ & $\begin{array}{l}\text { Recovery on self medication } \\
\text { a.Frequency of malaria infection } \\
\text { b.Number of subjects affected. }\end{array}$ & $\begin{array}{l}1(25) \\
2 x \\
2(50)\end{array}$ & $\begin{array}{l}7(87.5) \\
1-3 x \\
6(75 \%)\end{array}$ & $\begin{array}{l}5(62.50) \\
2-12 x \\
5(62.50)\end{array}$ & $\begin{array}{l}4(86) \\
2-3 x \\
2(10 \%)\end{array}$ & $\begin{array}{l}16(64 \%) \\
15(60 \%)\end{array}$ \\
\hline 4 & $\begin{array}{l}\text { Method of treatment: } \\
\text { a-Use of orthodox treatment } \\
\text { b--Use of traditional treatment } \\
\text { c-Use of Herbal treatment } \\
\text { - }\end{array}$ & $3(75)$ & $\begin{array}{l}8(100 \%) \\
0 \%\end{array}$ & $\begin{array}{l}8(100 \%) \\
0 \% \\
0 \%\end{array}$ & $\begin{array}{l}5(100 \%) \\
- \\
-\end{array}$ & $24(96 \%)$ \\
\hline \multirow[t]{6}{*}{5} & Prohyplaxic against malaria: & & & & & \\
\hline & $\begin{array}{l}\text { a-Use of prophylaxic drugs } \\
\text { b-Knowledge of ITN } \\
\text { c-Use of ITN }\end{array}$ & $\begin{array}{l}1(25) \\
1(25) \\
0 \%\end{array}$ & $\begin{array}{l}5(62.50) \\
4(50 \%) \\
1(12.5)\end{array}$ & $\begin{array}{l}1(12.5) \\
7(87.5) \\
3(37.5)\end{array}$ & $\begin{array}{l}1(20 \%) \\
5(100 \%) \\
2(40 \%)\end{array}$ & $\begin{array}{l}8(32 \%) \\
17(68 \%) \\
6(24 \%)\end{array}$ \\
\hline & d-Re-impregnation of net & 0 & 0 & 0 & 0 & \\
\hline & e-Use of insecticide & $2(50)$ & $6(75 \%)$ & $6(75 \%)$ & $5(100 \%)$ & $19(76 \%)$ \\
\hline & Immunization against: & & & & & \\
\hline & $\begin{array}{l}\text { f-Malaria infection } \\
\text { g-:Typhoid fever }\end{array}$ & $\begin{array}{l}0 \% \\
1(25)\end{array}$ & $\begin{array}{l}0 \% \\
1(12.5)\end{array}$ & $\begin{array}{l}0 \% \\
0 \%\end{array}$ & $\begin{array}{l}1(20 \%) \\
2(40 \%)\end{array}$ & $\begin{array}{l}1(4 \%) \\
3(12 \%)\end{array}$ \\
\hline \multirow[t]{2}{*}{6} & Presence of breeding places: & & & & & \\
\hline & $\begin{array}{l}\text { a-Water logged } \\
\text { b-Swamp } \\
\text { c-Open drainage } \\
\text { d-Bush }\end{array}$ & $\begin{array}{l}1(25) \\
1(25) \\
\\
0(0 \%)\end{array}$ & $\begin{array}{l}2(25) \\
2(25) \\
2(25) \\
0\end{array}$ & $\begin{array}{l}3(37.5) \\
2(12.5) \\
3(37.5) \\
-\end{array}$ & $\begin{array}{l}5(100 \%) \\
2(40 \%) \\
1(20 \%) \\
0.0 \%\end{array}$ & $\begin{array}{l}11(44 \%) \\
6(24 \%) \\
7(28 \%)\end{array}$ \\
\hline 7 & Absence from work due to malaria & 0 & $\begin{array}{l}\text { 3days } \\
1(12.5)\end{array}$ & 2days & $\begin{array}{l}\text { 2days } \\
1(20)\end{array}$ & $2(8 \%)$ \\
\hline 8 & Previous typhoid infection & $1(25)$ & $3(37.5)$ & $0 \%$ & $3(60 \%)$ & $7(28 \%)$ \\
\hline
\end{tabular}

Five recovered on self medication, while 2 others claimed to recover sometimes. 
Table 9: Health Related Activities Undertaken By Male Staff Groups Aged 31-60years.

\begin{tabular}{|c|c|c|c|c|c|}
\hline \multicolumn{2}{|c|}{ Parameter } & \multicolumn{3}{|c|}{ Age groups } & \multirow{2}{*}{$\begin{array}{ll}\text { Total } & \\
50-60 & \\
5(100) \quad 37 \\
\end{array}$} \\
\hline \multicolumn{2}{|c|}{ No of subject in group (\%) } & $\begin{array}{l}31-40 \\
16(100 \%) \\
\end{array}$ & $\begin{array}{l}4-50 \\
10(100 \%) \\
\end{array}$ & $\begin{array}{l}21-30 \\
6(100 \%) \\
\end{array}$ & \\
\hline 1 & $\begin{array}{l}\text { Visit to medical centre: } \\
\text { aWhen ill } \\
\text { b During malaria infection } \\
\text { c When other medication fails }\end{array}$ & $\begin{array}{l}10(62.5) \\
6(57.5) \\
4(25)\end{array}$ & $\begin{array}{l}4(40) \\
3(30) \\
1(10)\end{array}$ & $\begin{array}{l}3(50) \\
2(33.33) \\
0(0)\end{array}$ & $\begin{array}{ll}4(80) & 21(56.76) \\
3(60) & 14(37.84) \\
1(20) & 6(16.22) \\
\end{array}$ \\
\hline 2 & Recovery on self medication & $13(81.25)$ & $6(60)$ & $5(83.33)$ & $4(80) 28(75.68)$ \\
\hline 3 & $\begin{array}{l}\text { a Frequency of malaria infection } \\
\text { (per annum) } \\
\text { b Number of subjects affected }\end{array}$ & $\begin{array}{l}2-4 x \\
10(62.5)\end{array}$ & $\begin{array}{l}2-6 x \\
7(70)\end{array}$ & & $\begin{array}{l}2 x \\
1(20) \quad 20(54.05)\end{array}$ \\
\hline 4 & Method of treatment & & & & \\
\hline & $\begin{array}{l}\text { a Use of orthodox treatment } \\
\text { b Use of traditional treatment } \\
\text { c Use herbal treatment }\end{array}$ & $\begin{array}{l}14(87.5) \\
1(6.25) \\
0(0)\end{array}$ & $\begin{array}{l}8(80) \\
1(10) \\
1(10)\end{array}$ & $\begin{array}{l}3(50) \\
1(16.67) \\
1(16.67)\end{array}$ & $\begin{array}{ll}5(100) & 30(81.08) \\
0(0) & 3(8.11 \%) \\
0(0) & 2(5.41) \\
\end{array}$ \\
\hline 5 & Prophylaxix against malaria & & & & \\
\hline & $\begin{array}{l}\text { a- Use of prophylaxis drugs } \\
\text { b- Knowledge of ITN } \\
\text { c- Use of ITN } \\
\text { d- Re-impregnation of net } \\
\text { e- Use of insecticide }\end{array}$ & $\begin{array}{l}9(56.25) \\
11(68.75) \\
5(31.25) \\
1(6.25) \\
10(62.5) \\
\end{array}$ & $\begin{array}{l}2(20) \\
7(70) \\
2(20) \\
(0) \\
5(50)\end{array}$ & $\begin{array}{l}3(50) \\
6(100) \\
0(0) \\
0(0) \\
3(50) \\
\end{array}$ & $\begin{array}{ll}3(60) & 17(54.95) \\
4(80) & 28(75.68) \\
0(0) & 7(18.92) \\
0(0) & 1(2.7) \\
4(80) & 22(59.46) \\
\end{array}$ \\
\hline & Immunization against & & & & \\
\hline & $\begin{array}{l}\text { f- Malaria infection } \\
\text { g- Typhoid fever }\end{array}$ & $\begin{array}{l}5(31.25) \\
2(12.5) \\
\end{array}$ & $\begin{array}{l}0(0) \\
2(20) \\
\end{array}$ & $\begin{array}{l}2(33.33) \\
0(0)\end{array}$ & $\begin{array}{ll}1(20) & 8(21.62) \\
0(0) & 2(5.41) \\
\end{array}$ \\
\hline & Presence of breeding places: & & & & \\
\hline & $\begin{array}{l}\text { a Water-logged } \\
\text { b Swamp } \\
\text { c Open drainage } \\
\text { d Bush }\end{array}$ & $\begin{array}{l}8(50) \\
2(12.5) \\
2(12.5) \\
2(12.5) \\
\end{array}$ & $\begin{array}{l}4(40) \\
1(10) \\
3(30) \\
1(10) \\
\end{array}$ & $\begin{array}{l}3(50) \\
1(16.67) \\
1(16.67) \\
0 .(0) \\
\end{array}$ & 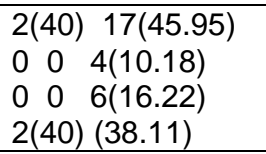 \\
\hline 7 & $\begin{array}{l}\text { Absence from work due to } \\
\text { malaria infection } \\
\text { Previous typhoid infection }\end{array}$ & $\begin{array}{l}\text { 1-7days } \\
3(18.75) \\
11(68.75)\end{array}$ & $\begin{array}{l}\text { 2days } \\
1(10) \\
3(30)\end{array}$ & $\begin{array}{l}\text { 3days } \\
1(16.67) \\
1(16.67)\end{array}$ & $\begin{array}{l}1(20) 6(16.22) \\
1(20) 16(43.24)\end{array}$ \\
\hline
\end{tabular}

Table 10: Mean ( \pm SD) Levels of serum electrolytes in various disease groups ( $\mathrm{mmol} / \mathrm{L})$.

\begin{tabular}{|l|l|l|l|l|l|}
\hline Parameters & Control & Malaria & Typhoid & Diabetes & Hypertension \\
\hline Number in group & 10 & 10 & 10 & 10 & 10 \\
\hline $\begin{array}{l}\text { Mean age } \\
\text { (years) }\end{array}$ & $50.4 \pm 5.81$ & $53.7 \pm 6.37$ & $50.3 \pm 5.65$ & $50.6 \pm 6.03$ & $53.2 \pm 6.25$ \\
\hline $\mathrm{Na}^{+}$ & $138.3 \pm 4.42$ & $127.0 \pm 2.26$ & $126 \pm 4.59$ & $142.3 \pm 3.94$ & $142.8 \pm 3.88$ \\
\hline $\mathrm{K}^{+}$ & $4.29 \pm 0.46$ & $3.4 \pm 0.41$ & $3.2 \pm 0.48$ & $3.39 \pm 0.35$ & $3.43 \pm 0.25$ \\
\hline $\mathrm{Cl}^{\mathrm{HCO}_{3}^{-}}$ & $100.2 \pm 4.02$ & $94.7 \pm 2.75$ & $93.6 \pm 2.27$ & $104.4 \pm 4.37$ & $102.4 \pm 3.86$ \\
\hline $\mathrm{Ca}^{2+}$ & $27.1 \pm 3.72$ & $23.1 \pm 3.10$ & $23.1 \pm 2.33$ & $22.8 \pm 2.14$ & $23.10 \pm 2.84$ \\
\hline $\mathrm{Pi}$ & $1.30 \pm 0.08$ & $1.30 \pm 0.05$ & $1.30 \pm 0.02$ & $1.32 \pm 0.02$ & $1.30 \pm 0.03$ \\
\hline
\end{tabular}


Agric. Biol. J. N. Am., 2012, 3(10): 413-426

Table 11: Mean ( \pm SD) Levels of serum biochemical parameters in various disease groups (mmol/L).

\begin{tabular}{|c|c|c|c|c|c|}
\hline Parameters & Control & Malaria & Typhoid & Diabetes & Hypertension \\
\hline $\begin{array}{l}\text { Number in } \\
\text { group }\end{array}$ & 10 & 10 & 10 & 10 & 10 \\
\hline $\begin{array}{c}\text { Mean age } \\
\text { (years) }\end{array}$ & $42.7 \pm 5.11$ & $40.8 \pm 5.03$ & $43.5 \pm 5.62$ & $44.2 \pm 5.79$ & $51.5 \pm 6.58$ \\
\hline Cholesterol & $0.61 \pm 1.06$ & $4.1 \pm 0.50$ & $5.15 \pm 0.46$ & $4.99 \pm 0.57$ & $3.56 \pm 0.71$ \\
\hline Albumin & $2.97 \pm 7.85$ & $22.9 \pm 0.34$ & $28.4 \pm 1.54$ & $27.18 \pm 2.25$ & $21.9 \pm 0.93$ \\
\hline Creatinine & $10.8 \pm 32.1$ & $138.7 \pm 3.23$ & $139.8 \pm 4.51$ & $137.6 \pm 6.61$ & $140.0 \pm 4.37$ \\
\hline Bilirubin & $5.29 \pm 5.44$ & $9.95 \pm 1.12$ & $17.38 \pm 0.40$ & $17.78 \pm 0.42$ & $11.08 \pm 1.62$ \\
\hline Uric acid & $47.22 \pm 73.81$ & $423.9 \pm 22.92$ & $383 \pm 45.48$ & $323.5 \pm 50.21$ & $423.8 \pm 25.69$ \\
\hline
\end{tabular}

\section{DISCUSSION}

The present study has determined the illness pattern in a Nigerian university. The most prevalent illnesses include malaria, upper respiratory tract infection, peptic ulcer, and V. candidiasis, among others. The following discussion reviews the number of the people affected by malaria infection alongside their responses on questionnaires based on health related activities. Hence the focus of the present work covers the pattern of frequencies of malaria infection and their implication. Also to be discussed are the results of mean $( \pm S D)$ comparisons/correlation coefficient of the illness patterns, as well as that of the biochemical parameters in the various disease states.

Pattern of Malaria Frequencies: The pattern of malaria infection showed that the frequencies of infection were highest between the months of March and June, with a pick frequency in April (Figs 1-5). The period - March to June, coincides with the rainy season which favours the springing-up of breeding places - swamps, bushes, stagnated drains and water-logs. Hence any intervention technique should cover these critical periods so as to properly stamp out the mosquito vectors that inhabit thereby.

Malaria, as the most prevalent of the illness cases (63.83 - 67.14\%) reported in our present work affected about 1083-1969 people yearly on campus $(1-5)$.

It was also observed that only about $30.43-31.58 \%$, $28.69-33.33 \%, 25-60 \%$ and $30-60 \%$ of the female students, male students, female staffs, and male staff groups respectively visited the health centre on account of malaria illness (tables 6 - 8). This meant that the above number of malaria cases who reported at the health centre yearly $(1083-1969)$ should be deemed as an understatement, and did not account for all the people who had malaria attack during the study period. The rest people (calculated as the difference between those who suffered the infection and those who reported sick at the medical centre for treatment), $29.92 \%$ of the 137 female subjects (table $6 ; 1 \mathrm{~b} \& 3 \mathrm{~b}$ ), $41.76 \%$ of the 158 male students (table $7 ; 1 b$ \& $3 b$ ), $36 \%$ of the 25 female staff (table $8 ; 1 b$ \& $3 b$ ), and $16.21 \%$ of 37 male staff (table $9 ; 1 b \& 3 b$ ), either sought for treatment elsewhere or resorted to self medication.

Also out of the female students group who participated, $61.31 \%$ claimed a frequency of one to eight bouts of malarial infection per annum, (table 6; $3 b)$. From the rest groups, the ratio $70.89 \%$ for the male students (table $7 ; 3 \mathrm{~b}$ ), $60 \%$ for the female staff (table $8 ; 3 \mathrm{~b}$ ), and $54.05 \%$ for the male staff were obtained. These evidences had further strengthened the fact that the statistics on malaria cases often reported from the health centers and subsequently published in literatures were merely a tip of the iceberg. The actual number of malaria cases could be well above what is currently reported in literatures. The same logic applies to the other medical cases. The number of people who use other unorthodox means of treatment are not always taken into account during such counts.

Implication of IIIness Pattern on Policy
Formulation/Planning, and Economic
Development: One implication of the above
consideration is that no meaningful health policy
could be effectively applied in the face of inadequate
health statistics. Policy formulation and planning are
necessary tools in curbing the problem of excesses
(in spending) or under-utilization of economic power
and resources. Projects that are under-funded will
sooner or later be abandoned, making the whole
process an exercise in futility. This could be true
when one considers the previous attempts to
eradicate malaria. For example half-hazard attempt


on the destruction of mosquito-breeding places would not completely stamp-out the mosquito vectors. One single bite from an infective female anopheles mosquito vector is enough to start the transmission circle all over and before long many people would have been affected. At the end of the day the "Roll Back Malaria 2020"project would be nothing but a mirage.

Malaria had been associated with poverty and poor economic development (Bredman et al, 2004). These scholars estimated that the economic cost due to malaria infection for Africa alone was equivalent to USD12 billion annually. Malaria had also been estimated to be responsible for high mortality rate of infants, young children and pregnant women, (Hoffman, 2004). The symptoms produced by the infection include fever, anaemia, loss of body weight, aches/pains, vomiting and sometimes diarrhea (WHO/CDC, 2003), which could culminate into undernutrition, and eventually high morbidity and mortality rates if not adequately treated.

Malaria and Undernutrition: The vicious relationship between infection and undernutrition in the developing countries of the world has log been recognized (Chandra, 1980). This relationship is strengthened by the fact that many children from the developing world are on boarder-line nutritional status such that further nutritional deprivation during periods of infection (with accompanying fever and anorexia) overwhelms the individual who then succumbs to nutritional diseases of various forms.

Under nutrition weakens the immunity and his ability to fight infection. The synergism that nutritional deficiencies increase the frequencies and severity of infections on one, and on the other hand that infection deepens nutritional deprivation suggest that the solution to the problem should not be limited to one single approach but encompass a multifaceted one which involves other sectors. Such multifaceted approach should include immunization, portable water supply, sanitation, health education, nutritional supplementation, agricultural production, economic development, breast feeding, maternal health and prevention of low birth weight. The present work reveals that some of these approaches, such as immunization and nutrient supplementation, are currently being undertaken but at a very low level. In addition our study suggests that if malaria infection is eradicated, then the illness/infection rate would have been reduced to nothing less than one half for the child, adult and pregnant mothers alike.
Incidence of Malaria: From the foregoing it does seem that the figures on the incidence of malaria infection published in many literatures could have been underestimated. The reason is that many a times such reports fail to take cognizance of those who used self medication or other unorthodox means such as herbal and traditional medication in disease treatment. For example literatures had reported a malaria incidence of 300 to 500 million cases per annum and a mortality rate of 2 to 3 million people yearly (Niikura et al, 2008; Hoffman, 2004; Okie, 2003). These estimations appear good but may be deemed low judging by our present calculations in a malaria endemic environment in the south-south region. Our present work suggests that malaria affects more than one half, $64.71 \%$, of the students' population on Niger Delta University campus. If this applies to other universities in the west and southern part of the country having similar terrain, the number of the Nigerian population affected would be so high.

Economic Implication of Malaria Infection: Our study estimates that malaria affects more than one half of the studied population. If by extension this applies to other parts of the country having similar terrain, then Nigeria with a population of about 160 million people, may have about 130 million of them at risk or being infected with malaria. This means that Nigeria would spend large part of her financial resources in combating malaria. These resources would have been re-invested into a more productive sector of the economy that would enhance economic development which in turn should lead to a better standard of living for the people. The issue of malaria should be tackled once and for all, so as to avoid wastage of scarce financial resources

Strategies to "Roll Back Malaria, 2020": In the past many strategies were put in place in order to wipe out malaria. For one reason or the other many of them lack the required supervision and enforcement necessary to see them to successful completion and sustainability. One such example is the introduction of insecticide-treated nets (ITN). From our present analysis, it was shown that the percentage of respondents who claimed knowledge of ITN was low and comprised mostly those within age-groups 11-20 and 51-60 (years), (tables 6-9; 5b). Also among those who claimed knowledge of ITN, only about $18-38 \%$ (less than one half the population) of them actually made use of the nets. Worse still, many of the users never re-impregnated nets all through the period of use (tables 6-9). 
Mean ( $\pm S D$ ) Comparison and Correlation coefficient Between Illness Patterns: Mean comparison by use of ANOVA showed a significant difference between the means of malaria frequencies in years 2011 and 2007, $(p<0.05)$. No significant difference was determined between the mean $( \pm S D)$ frequencies in 2007 and those from the rest years. Also no significant difference was obtained in the means $( \pm S)$ of typhoid frequencies nor in those of URTI. On the other hand, results of correlation showed a significant positive correlation between malaria and typhoid, $r=0.587,0.970$ and 0.759 $(p<0.05)$, for the years 2009, 2010 and 2011, respectively. There was no reported typhoid fever case in the year 2008; while the correlation coefficient for 2007 was not significant $r=-0.245$ $p>0.05)$. Positive correlation was also obtain between the frequencies of malaria and URTI, $r=0.806$, $0.874, \quad 0.849$ and $0.759(p<0.05)$, for the years 2007, 2008, 2009 and 2011 respectively.

Similarly the data for the biochemical parameter $\mathrm{HCO}_{3}$ \& $\mathrm{Cl}^{-}, \mathrm{P} \& \mathrm{Ca}$, albumin \& cholesterol, and $\mathrm{Na}$ \& $\mathrm{K}$ were positively correlated. Correlation coefficient was used to assess for any statistical relationship or interplay between the biochemical parameters. Change in the levels and interrelation between analytes probably occurs depending on the extent of pathology, and this could possibly account for lack of uniformity in previous work (Madukosiri and Adoga, 2007; Sirkorski et al, 1988). Correlation determined between analytes could represent interaction in metabolism and normal homeostatic interplay between them. Correlation among these elements had also been demonstrated in plasma and in serum, ( Madukosiri and Adoga 2007; Sirkoski et al, 1988). Lack of statistical correlation was then deemed as absence of association or interaction in metabolism. However when fully developed and its usefulness fully understood, the use of statistics in determination of association between metabolites could serve as a useful tool in predicting their levels as well as featuring as a signaling sensor in the determination of disease prognosis/progression in clinical setups, particularly where laboratory facilities are scanty or inadequate.

Positive Action Against Malaria: Action against malaria should include elimination of mosquito vectors by extermination of breeding places. Immunization or prophylaxis management should be enforced in schools, covering both the primary and tertiary levels. This would ensure smooth academic programme for students and improvement of man- hour for workers. Students who fail to show evidence of malaria prophylaxis may be denied registration. For the working class they may stand the chance of denial of privileges if they fail to avail themselves of the necessary prophylaxis against malaria. The use of ITN should be enforced and demonstrated at regular intervals, not just in a sporadic manner. Elimination of malaria will definitely impact positively on the academic performance of students and by extension on the level of expertise, since absenteeism from classes or jobs due to morbidity would then be minimal. Apart from impacting positively on education it should affect economic growth and development, because the resources which would have been spent on malaria treatment, drugs and insecticides purchase, should then be directed to human resources and production sectors. Until this disease is controlled and eradicated, efforts to improve health, economic development and reduction in poverty level may prove futile.

\section{CONCLUSION}

The present work has determined the illness pattern and relationship between the prevalence of malaria and other infections such as typhoid, URTI, peptic ulcer, and v. candidiasis, (in that order), from year 2007 - 2011. Malaria was found to be the most prevalent illness with the highest frequency occurring around the months of March to June - months which coincide with the rainy season when breeding places spring-up.

Evidence was presented to show that some published statistics on malaria might have been underestimated. Those who suffered malaria and who used self medication or other unorthodox means, such as herbal or traditional medication for treatment, were not often accounted for in such data.

Mean comparison by use of ANOVA showed a significant increase in frequency of malaria infection from year 2007 to 2011 despite measures already put in place to 'Roll back malaria' come the year 2020.

It is no gain saying that malaria infection has hitherto remained an endemic disease in Nigeria and has caused untold hardship to its people. It has been linked with high morbidity and mortality rate across age groups and in pregnancy .The economic waste incurred by the infection is inestimable. This scenario could be summed up in the words of the poem;"

"I hate you mosquito!..

Ever since you continue to torment man, And to ensure you are kept at bay, More and more chemicals are made; 
But you develop immunity against them all... I dread your irritating voice when you sing I hate the needle-like piercing of your sting I'd rather listen to the orchestra of frogs at night

Than behold you within my sight

And oh! what an insult!

Mortals hold world conference to discuss

you...

You multiply before the morning dew

And when it's rainy

You bring sickness and death to many...

It's past eleven

You're already roaring like the devil

Set to steal blood, kill some and destroy

others...

l've got my mosquito net..."

Who wouldn't let mortals rest...."

(Afiesimama and Afiesimama, 2005).

\section{RECOMMENDATION}

The importance of evidence-based recommendation in health policy formulation cannot be overemphasized. It cuts down excesses and minimizes losses in project implementation. The present work has attempted to generate evidencebased recommendation which should find application on policy formulation. It is likely that the frequency of malaria infection and its menace on health, and by extension on economic development, are often undermined and underestimated. Malaria still remains a major endemic disease with the highest frequency of occurrence on campus - the frequency which was highest within the month of April.

The menace of malaria is unlikely to see its end in the nearest future, while the speculated economic development will remain a mirage if nothing is done to nib this malady from the bud. Definite strides must be made in the right direction - elimination of breeding places (particularly water-logs and open drainage), and enforcement of immunization or prophylaxis therapy as the case may be. Enlightenment campaign is unavoidable and must demonstrate the use and usefulness of ITN. In schools, if possible, students should show evidence of immunization or prophylaxis against malaria before they are allowed to register. This could represent the "extra mile" in the bid to wipe out this endemic disease from our environment

\section{ACKNOWLEDGEMENT}

Our profound gratitude goes to the Federal Government of Nigeria through its operative,
TETFUND (Tertiary Education Trust Fund), whose sponsorship made it possible for this paper to be presented at the conference of International Union of Biochemistry and Molecular Biology / Federation of European Biochemists (IUBMB/FEBS), Sevilla Spain, 2012. We also acknowledge all those who contributed one way or the other to the completion of this research. We are particularly grateful to the Director of Health Services, Dr E. T Omu, for his cooperation during the sampling and data collation; and to Dr E Samuel, Head of Department of Economics, Niger Delta University, Bayelsa, for his invaluable advice which contributed to the success of the present project

\section{REFERENCES}

Baridan D.M (1990). Research Method in Administrative Science. Belk Publishers, Port Hercourt, Nigeria.

Breman J. G, Alilio, M.S. and Mills A, (2004). Conquering the intolerable burden of malaria: What's new, what needed, a summary. Am. J. Trop Med. Hyg. 71 (2suppl). 1 - 5.

Burtis Carl A; and Ashwood Edward R, (2001).Tiez Fundamentals of Clinical Chemistry ( $5^{\text {th }}$ edition). Elsevier, New Delhi, 1091p.

Chandra R.K (1980). Immunology of Nutritional Disorders. Edward Arnold Ltd, Badford Square, London, 110p.

Hoffman S., (2004). Save the children. Nature 430; 940941

Lerner K.L, Lerner B.W, (2003). World of Microbiology and Immunology. Vol.1 and 2: 5,7,25,185-189.

Madukosiri C.H; Adoga G.I, (2011). Elemental status of pregnant and lactating women in Bassa LGA of Plateau state, Nigeria. Pakistan Journal of Nutrition, 10(5), $401-404$.

Niikura M, Kamiya S, Kiyoshi K, Fumie k, (2008). Coinfection with Non-lethal Murine Malaria parasites suppresses pathogenesis caused by plasmodium. J. Immunol, 180: 6877-6884.

Okie S. (2005). Betting on a malaria vaccine. N. Engl. J. Med. 353: 1877-1881.

Sirkorski R; Paszkowski, T; Milart, P; Radomanski, T; Szkoda, J. (1988). Intrapartum levels of tragic metals in maternal blood in relation to unbilical cord blood values: lead, iron, copper, zinc. Int. J. Gynae and Obs. $26,213-221$.

WHO/CDC, (2003). Manual for Laboratory identification and Antimicrobial susceptibility Testing of Bacterial Pathogens of Public Health importance in Developing World, $103-115$. 